\title{
Interacción entre defensas costeras artificiales en playas arenosas y perturbaciones naturales de gran escala: el terremoto del $27 \mathrm{~F}$ de 2010 en la costa del centro-sur de Chile $^{1}$
}

\author{
Eduardo Jaramillo², Mario Manzano ${ }^{3}$, Gonzalo Morales ${ }^{4}$ \\ y Carlos Velásquez ${ }^{5}$
}

\begin{abstract}
RESUMEN
Durante los años 2010 y 2011, estudiamos los efectos del levantamiento costero producto del terremoto del $27 \mathrm{~F}$ sobre el paisaje físico y la macrofauna de playas arenosas, ubicadas frente a defensas costeras artificiales en la península de Arauco y alrededores. El ancho promedio de las playas durante el periodo posterior al $27 \mathrm{~F}$ fue hasta 12,5 veces mayor que los anchos observados antes del terremoto y la pendiente de las playas levantadas disminuyó. En playas que tenían defensas costeras ubicadas cerca del nivel de marea alta, el espacio restaurado en los niveles superiores y medios del intermareal (previamente ocupados por murallas y revestimientos rocosos), fue rápidamente colonizado por crustáceos que habitan niveles intermareales similares en playas sin defensas costeras artificiales. Estudios llevados a cabo luego de la ocurrencia de grandes perturbaciones, son relevantes para aumentar el conocimiento sobre las interacciones entre fenómenos naturales de gran escala y la infraestructura costera artificial.
\end{abstract}

Palabras clave: Playas de arena, macrofauna, levantamiento continental, centro-sur de Chile.

\begin{abstract}
During 2010 and 2011, we studied the effects of the continental uplift that resulted from the earthquake of $27 \mathrm{~F}$ on the physical landscape and the macrofauna of sandy beaches located in front of coastal armoring along Península de Arauco and nearby areas. The mean width of uplifted beaches during the period following $27 \mathrm{~F}$ was up to 12,5 times higher than beach widths observed before the earthquake and the beach face slope of uplifted beaches decreased. At those beaches that had coastal armoring located closer to high tides before the earthquake, the restored upper and mid shore beach levels (previously occupied by seawalls and rocky revetments) were rapidly colonized by crustacean species, which typically inhabit at similar intertidal levels of unarmored beaches of Chile. Field samplings carried out promptly after large scale disturbances have occurred, are relevant to increase knowledge on the interaction between that natural phenomenon's and coastal artificial infrastructure.
\end{abstract}

Key words: Sandy beaches, macrofauna, continental uplift, south central Chile.

\footnotetext{
Estudio financiado por Proyectos FONDECYT $\mathrm{N}^{\circ}$ 1090650 y $N^{\circ} 1121043$. Los autores agradecen a Marcia González por el trabajo de laboratorio, consistente en el sorteo de muestras faunísticas y reconocimiento de las especies de crustáceos, y a Joaquín Sobell (Valdivia) por la elaboración de algunas figuras. Artículo recibido el 28 de agosto de 2012 aceptado el 15 de septiembre de 2012 y corregido el 30 de septiembre de 2012 .
}

2 Instituto de Ciencias Ambientales y Evolutivas, Facultad de Ciencias, Universidad Austral de Chile (Chile).E-mail: edojaramillo@gmail.com

3 Ministerio de Obras Públicas (Chile). E-mail: mario.a.manzano.c@gmail.com

4 Biólogo Marino. E-mail: morales.gonzalo87@gmail.com

5 Instituto de Ciencias Ambientales y Evolutivas, Facultad de Ciencias, Universidad Austral de Chile (Chile). E-mail: cfvelasque@gmail.com 
Los grandes terremotos $(\mathrm{Mw}>8,5)$ que ocurren a lo largo de márgenes costeros ubicados en zonas tectónicas de subducción, resultan en levantamiento o subsidencia continental, lo que afecta notoriamente el paisaje litoral, incluyendo el hábitat físico y la biota bentónica del mismo (e.g. Plafker, 1965; Plafker \& Savage, 1970; Ortlieb et al., 1996a, 1996b; Castilla, 1988; Boggs \& Shephard, 1999; Castilla et al., 2010; Farías et al., 2010; Vargas et al., 2011; Jaramillo et al., 2012). Charles Darwin (1851) fue uno de los primeros en describir tales cambios; para ello se basó en las mediciones realizadas por él mismo en la costa de la península de Arauco, y por Robert Fitz Roy en la costa rocosa de la isla Santa María (circa $37^{\circ} \mathrm{S}$ ), quien midió alzamientos de hasta 2,4-3,0 m, basado en las distribuciones verticales de restos muertos de moluscos bivalvos de la familia Mytilidae.

La mayor parte de los estudios realizados acerca de los efectos ecológicos de las deformaciones costeras causadas por terremotos en zonas activas de subducción tectónica, están basados en la distribución vertical de la fauna y flora bentónica que ocurre sobre sustratos rocosos, siendo escasos los estudios tendientes a evaluar el efecto de esas deformaciones sobre las características físicas y biológicas de zonas arenosas intermareales (Jaramillo et al., 2012), las cuales representan cerca del $75 \%$ de la costa mundial libre de hielos (Brown, 2001).

Durante enero del año 2010, se muestrearon playas arenosas de la costa de las regiones del Maule y Biobío con el objetivo de evaluar el efecto de defensas costeras artificiales como murallas de concreto y revestimientos rocosos, sobre la estructura física y macrofauna intermareal de esas playas (Jaramillo, 2011). La construcción de tales estructuras ha sido por siglos una respuesta societaria a la erosión y retroceso de la línea de costa (Nordstrom, 2000; Charlier et al., 2005). Sin embargo, la instalación de tales defensas costeras origina efectos locales como erosión de arena en áreas cercanas o en la base de estas estructuras (e.g. Krauss \& McDougal, 1996; Griggs, 2005a, 2005b), aumento en la frecuencia con que las olas interactúan con la línea de costa y reducción de parte de la zona intermareal, un hábitat costero ocupado por una fauna de inverte- brados abundante y diversa (e.g. McLachlan \& Jaramillo, 1995; Jaramillo 2001). Estudios llevados a cabo en playas arenosas de la costa de California y del centro-sur de Chile, muestran que tal reducción resulta en la eliminación de la fauna de invertebrados bentónicos que habita los niveles superiores de esas playas (Dugan \& Hubbard, 2006; Dugan et al., 2008; Jaramillo, 2011).

Aquí el terremoto del 27 de febrero 2010 (27F) resultó en levantamientos continentales de alrededor de dos metros en la península de Arauco y alrededores (circa $37-38^{\circ}$ S) (e.g. Vargas et al., 2011; Jaramillo et al., 2012), lo que resultó en mortandades masivas de la flora y fauna bentónica del litoral rocoso afectado (e.g. Castilla et al., 2010). En este estudio se evalúa el efecto de ese levantamiento sobre el paisaje físico y las abundancias poblacionales de la macrofauna intermareal de playas arenosas ubicadas frente a defensas costeras artificiales que con anterioridad al $27 \mathrm{~F}$, tenían diferentes grados de interacción con olas y mareas durante marea alta. Esto con el objetivo de evaluar la interacción entre ese desarrollo costero y perturbaciones naturales de gran escala, como lo fue el terremoto del $27 \mathrm{~F}$.

\section{Materiales y métodos}

Los sitios de muestreo se ubicaron en la península de Arauco y áreas cercanas, la zona costera con mayor levantamiento continental (c 2 m; Jaramillo et al., 2012) a lo largo de la zona de fractura de este terremoto. Dos de las playas estudiadas tienen defensas costeras artificiales que con anterioridad al $27 \mathrm{~F}$, interactuaban directamente con las olas durante marea alta (punta Lavapié y Llico, en la Figura $N^{\circ} 1$ ), a la vez que una de ellas (Lebu, en Figura $N^{\circ} 1$ ) tiene una muralla que no interactuaba directamente con las olas durante ese período mareal, salvo durante períodos de temporales y marejadas. Los resultados que se presentan a continuación corresponden a muestreos realizados durante enero, marzo, julio, septiembre y diciembre 2010 y marzo, julio y noviembre 2011.

Todos los muestreos se realizaron durante mareas de sicigia, período durante el cual la zona intermareal es más ancha, debido a que 
las diferencias entre la marea baja y marea alta es mayor. Se midió el ancho y pendiente de cada sitio de muestreo sobre cuatro transectos, separados por $5 \mathrm{~m}$ y extendidos entre el límite posterior de la playa (definido por la base de las defensas costeras artificiales) y el nivel de la marea baja. El ancho de la zona de muestreo fue la distancia entre esos límites, a la vez que la pendiente de la playa se expresa como $1 / x$ donde $x$ fue la distancia en metros a la cual se encuentra una diferencia de altura de $1 \mathrm{~m}$ entre dos niveles intermareales consecutivos ( $c f$. Emery 1961). Es decir, valores más altos de $\mathrm{x}$ corresponden a playas más planas o de menor inclinación.

Se muestreó a lo largo de cada uno de los cuatro transectos mencionados anteriormente y en las tres zonas faunísticas que ocurren a lo ancho del intermareal de las playas arenosas del centro-sur de Chile (McLachlan \& Jaramillo, 1995; Jaramillo, 2001) y que están primariamente representadas por crustáceos, es decir: i) zona superior, ocupada primariamente por el anfípodo talítrido Orchestoidea tuberculata Nicolet y generalmente extendida entre las dunas o cortes naturales posteriores de las playas y el nivel de la marea alta; ii) zona media, ocupada por los isópodos cirolánidos Excirolana braziliensis Richardson y Excirolana hirsuticauda Menzies y extendida entre el nivel de marea alta y la línea de efluente o límite superior del espejo de agua;

Figura $\mathrm{N}^{0} 1$

Ubicación de los sitios de estudio en la península de Arauco y alrededores, centro-sur de Chile.

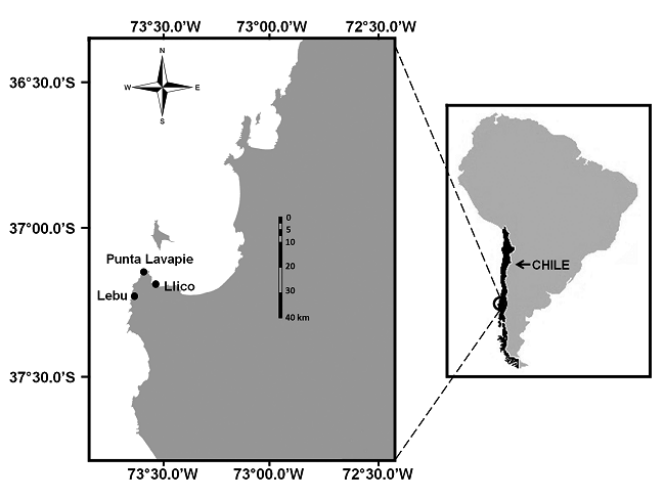

Fuente: Elaborado por Gonzalo Morales. y iii) zona inferior, ocupada primariamente por el decápodo anomuro Emerita analoga Stimpson y extendida entre la línea de efluente y el nivel más bajo de la marea baja (Figura $N^{\circ} 2$ ). Se recolectaron cinco muestras de arena a intervalos regulares en cada una de las zonas faunísticas mencionadas anteriormente. Para ello se utilizó un cilindro metálico de $10 \mathrm{~cm}$ de diámetro enterrado a una profundidad de $30 \mathrm{~cm}$ en el sustrato. Las cinco muestras recolectadas en cada zona, se juntaron para ser filtradas a través de un colador con malla de 1.000 micrones. Los organismos retenidos en la malla se conservaron en agua de mar-formalina $(10 \%)$ hasta posterior análisis en el laboratorio.

\section{Efectos del levantamiento continental sobre el paisaje físico y de la macrofauna intermareal de playas arenosas}

\section{El paisaje físico}

Antes del 27F, la muralla de concreto de punta Lavapié ocupaba gran parte del intermareal de la playa arenosa ubicada frente a la misma; el ancho de ese intermareal era de aproximadamente $12 \mathrm{~m}$ durante marea baja (Figura No 3a). Durante marzo de 2010, el ancho de la playa fue de $21 \mathrm{~m}$ (cerca de dos veces más), evidenciándose además la aparición de una serie de roqueríos que con anterioridad al terremoto estaban bajo el agua y que ahora limitan los niveles inferiores de esta playa (Figura $N^{\circ} 6$ ). Durante julio, septiembre y diciembre 2010, el ancho de la playa varió entre 24 y 32 m (hasta 2,0-2,7 veces más que el ancho estimado con anterioridad al 27F; Figura $N^{\circ} 3 \mathrm{~b}$ ). Los muestreos realizados durante el año 2011, evidencian mayores anchos de playa (55-68 m o 4,6-5,7 veces más intermareal que durante enero 2010; Figura $\mathrm{N}^{\circ} 3 \mathrm{~b}$ ), lo que probablemente es el reflejo de un activo proceso de depositación de arena favorecido por la presencia de los roqueríos emergidos luego del 27F. Coincidentalmente con el aumento observado en el ancho de la playa con posteridad al terremoto, se observó disminución de la pendiente de la misma, especialmente durante el año 2011 (2,7-3,5 veces más plana que durante enero 2010; Figura $N^{\circ} 3 b$ ). 
Figura $\mathrm{N}^{\circ} 2$.

Esquema de zonación de la macrofauna de crustáceos que ocurre a lo ancho de la zona intermareal de playas arenosas del centro-sur de Chile. Se indica el nivel de marea alta (MA), línea de efluente (LE) o límite superior del espejo de agua y el nivel de marea baja (MB). Los crustáceos son los anfípodos talítridos (Orchestoidea tuberculata o pulga de mar) que ocurren alrededor de MA o parte superior del intermareal, los isópodos cirolánidos Excirolana braziliensis y Excirolana hirsuticauda entre MA y LE o parte media del intermareal y los decápodos anomuros (Emerita analoga o chanchito de mar) desde la LE hacia la parte inferior de la playa.

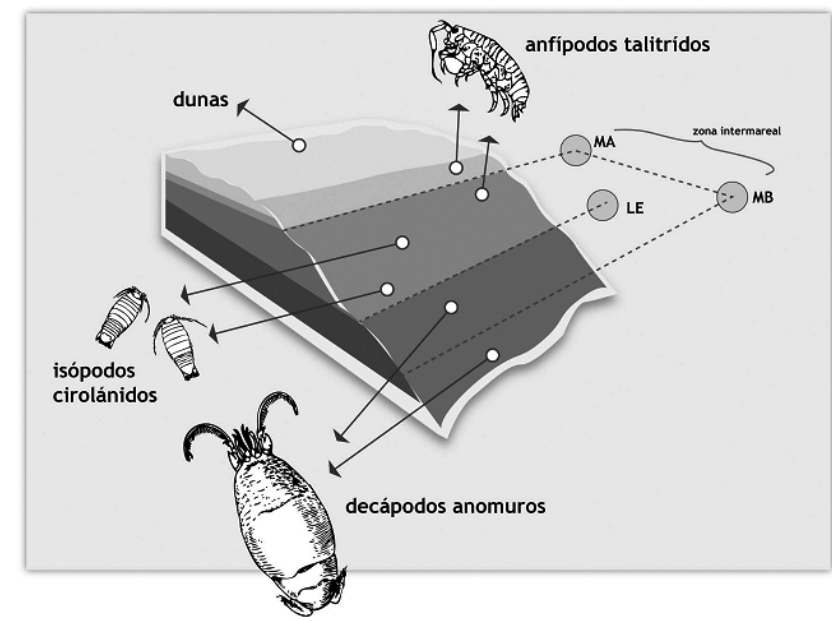

Fuente: Datos aportados por Eduardo Jaramillo. Esquema elaborado por Joaquín Sobell.

Durante enero de 2010, la playa de Llico, ubicada frente al revestimiento rocoso, era muy angosta, solo $8 \mathrm{~m}$ (Figura $\mathrm{N}^{\circ} 4 \mathrm{a}$ ). Las mediciones realizadas en marzo 2010 muestran que el ancho de la misma fue de $70 \mathrm{~m}$, lo que equivale a un aumento de 8,8 veces con respecto al muestreo de enero. Durante el resto de los muestreos del año 2010 y 2011, el ancho de la playa fue similar: 80-100 m, lo que equivale a 10,0-12,5 veces más que el ancho estimado con anterioridad al terremoto del 27F (Figura $N^{\circ} 4 \mathrm{~b}$ ). Durante marzo y julio de 2011, se midieron las menores inclinaciones de la playa, i.e., durante esos meses esta fue 2,3-2,5 veces más plana que durante enero 2010 (Figura No 4b). Durante el resto del período de estudio la playa fue $1,5-2,1$ veces más plana que con anterioridad al $27 \mathrm{~F}$ (Figura $N^{\circ} 4 \mathrm{~b}$ ).

Durante enero 2010, el ancho de la playa de Lebu era de 55 m (Figura $N^{\circ} 5 a$ ); un mes después (marzo 2010) ese ancho aumentó a $130 \mathrm{~m}$, lo que equivale a un incremento de 2,4 veces con respecto a enero (Figura 5b).
Durante julio y septiembre 2010, el ancho de la playa fue similar $(130$ y 120 m, respectivamente); durante diciembre 2010 y marzo 2011 se alcanzaron los valores máximos (162 y 145 $\mathrm{m})$, lo que equivalió a 2,6-2,9 veces el ancho medido con anterioridad al $27 \mathrm{~F}$ (Figura $\mathrm{N}^{\circ}$ $5 b$ ). Inmediatamente después del 27F (marzo 2010), la playa fue 1,6 veces más plana que con anterioridad al mismo, situación que se mantuvo relativamente constante a través del resto del período de estudio (Figura 5b).

\section{La macrofauna de las playas}

La Figura $N^{\circ} 6$ muestra que antes del terremoto la playa de punta Lavapié era ocupada solo por el crustáceo Emerita analoga, organismo típico de los niveles inferiores de las playas arenosas de Chile. Como consecuencia del 27F, la playa se levantó y su ancho aumentó (Figura $N^{\circ} 3$ ). Como mencionado anteriormente, se descubrió además una serie de roqueríos que con anterioridad al $27 \mathrm{~F}$ estaban siempre bajo el agua y que ahora, por estar emergidos, impiden que las 
Figura $\mathrm{N}^{\circ} 3$

Variabilidad estacional en la topografía de la playa arenosa ubicada frente a la muralla de concreto de punta Lavapié (a) y en el ancho y pendiente de la misma (b). Los números sobre los puntos son los radios entre los valores medidos con posterioridad al $27 \mathrm{~F} /$ valores estimados con anterioridad

al terremoto.
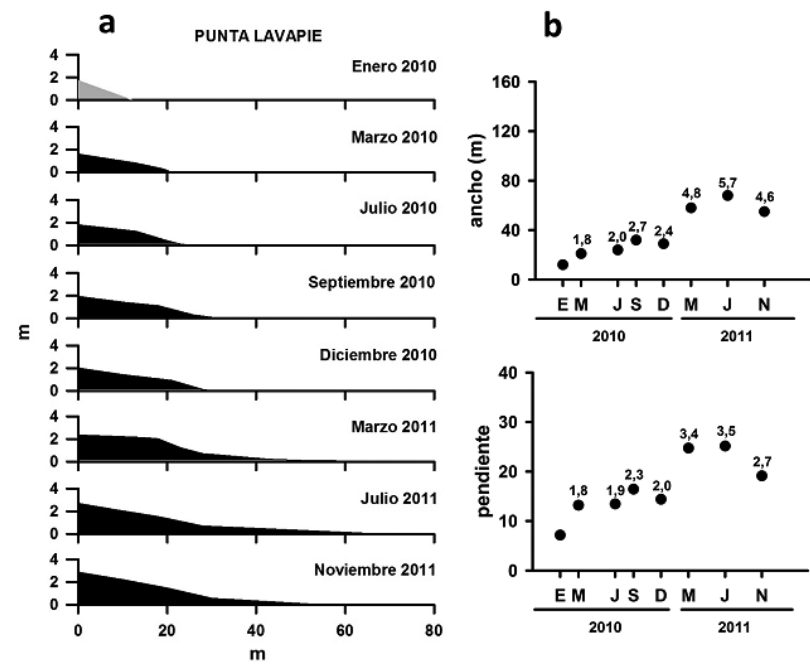

Fuente: Datos aportados por Eduardo Jaramillo. Esquema elaborado por Gonzalo Morales.

Figura $\mathrm{N}^{\circ} 4$

Variabilidad estacional en la topografía de la playa arenosa ubicada frente al revestimiento rocoso de Llico (a) y en el ancho y pendiente de la misma (b). Los números sobre los puntos son los radios entre los valores medidos con posterioridad al $27 \mathrm{~F} /$ valores estimados con anterioridad al terremoto.

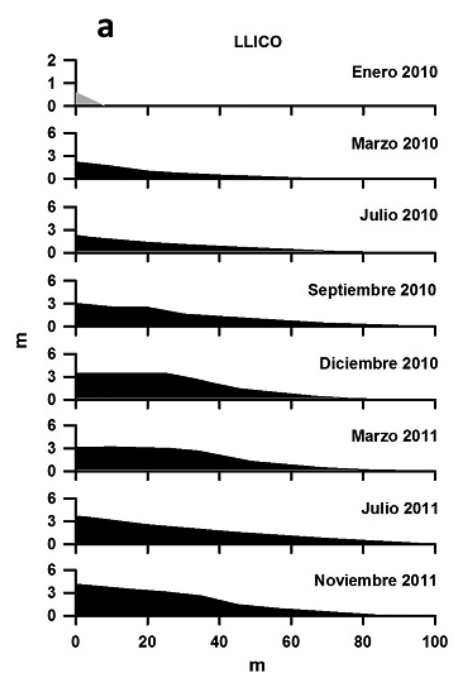

\section{b}
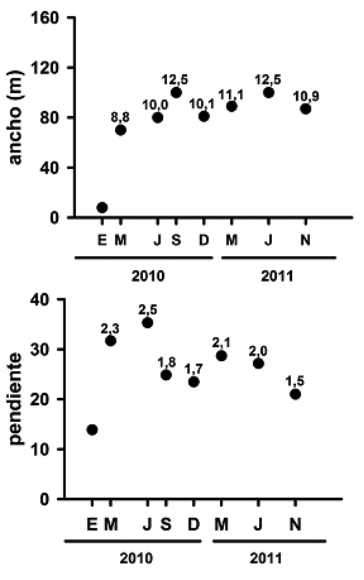

Fuente: Datos aportados por Eduardo Jaramillo. Esquema elaborado por Gonzalo Morales. 
Figura $\mathrm{N}^{\circ} 5$

Variabilidad estacional en la topografía de la playa arenosa ubicada frente a la muralla de concreto de Lebu (a) y en el ancho y pendiente de la misma (b). Los números sobre los puntos son los radios entre los valores medidos con posterioridad al $27 \mathrm{~F} /$ valores estimados con anterioridad al terremoto.
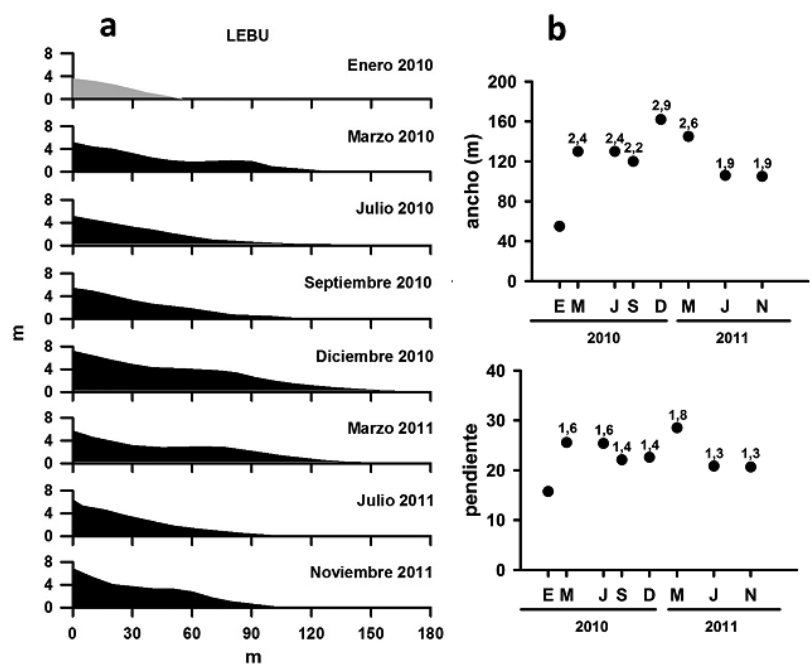

Fuente: Datos aportados por Eduardo Jaramillo. Esquema elaborado por Gonzalo Morales.

olas mojen la playa durante la marea baja. Esto hace que el contenido de agua en la arena haya disminuido, lo que puede explicar la desaparición de Emerita analoga (especie que ocurre en arenas con alto contenido de agua) durante la mayor parte de este estudio. Cinco y doce meses después de ocurrido el terremoto (julio de 2010 y marzo 2011, respectivamente), comienzan a aparecer en el nuevo espacio de playa restaurado frente a la muraIla, organismos típicos de los niveles medios medios (Excirolana braziliensis y Excirolana hirsuticauda) y superiores (Orchestoidea tuberculata) (Figura $\mathrm{N}^{\circ}$ 6) de playas que no han sido intervenidas con defensas costeras artificiales en playas arenosas del centro-sur de Chile. La Figura $N^{\circ} 6$ también muestra que durante los muestreos de julio y noviembre 2011 no se recolectaron isópodos cirolánidos en esta playa.

En Llico ocurrió algo similar a lo observado en punta Lavapié; antes del terremoto, la playa ubicada frente al revestimiento rocoso era ocupada solamente por Emerita analoga (Figura $N^{\circ} 7$ ). Luego del 27F (marzo 2010), el ancho de la zona intermareal aumentó (Fi- gura $\left.N^{\circ} 4 b\right)$ y especies típicas de los niveles superiores y medios de playas sin defensas costeras artificiales o no intervenidas (anfípodos talítridos e isópodos cirolánidos), comenzaron a ocupar el nuevo espacio restaurado debido al levantamiento continental (Figura $\mathrm{N}^{\circ} 7$ ).

La situación de la playa de Lebu se muestra en la Figura $N^{\circ}$ 8. Con anterioridad al 27F, la muralla presente en la misma se ubicaba bastante alejada de donde habitualmente llegaban las olas; es decir, la interacción entre la muralla, olas y mareas altas era baja. Antes del terremoto, esta playa albergaba todas las especies típicas que habitan la zona intermareal de playas arenosas del centro-sur de Chile carentes de defensas costeras artificiales. Esto se repite luego del terremoto; aun cuando las abundancias de Emerita analoga disminuyeron luego del $27 \mathrm{~F}$, debido al efecto del tsunami que alcanzó hasta los niveles superiores de esta playa produciendo mortalidades masivas de este crustáceo (Jaramillo et al., 2012). 
Figura $\mathrm{N}^{\circ} 6$

Variabilidad estacional en la composición de la macrofauna de crustáceos de la playa arenosa ubicada frente a la muralla de concreto de punta Lavapié.

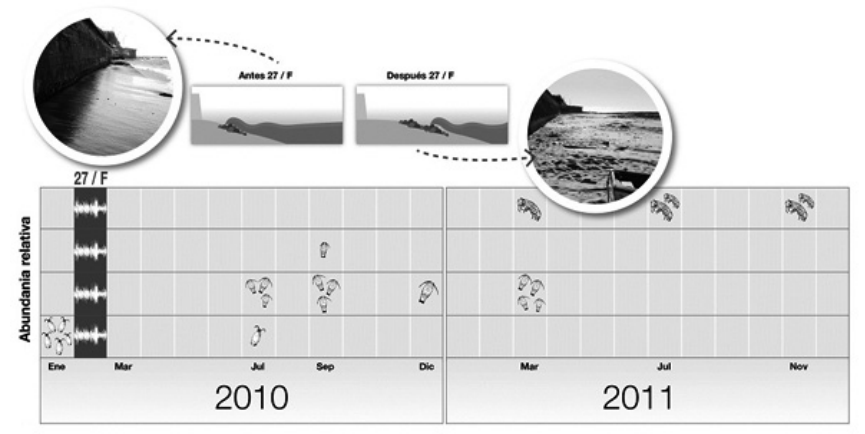

Fuente: Datos aportados por Eduardo Jaramillo. Esquema elaborado por Joaquín Sobell.

Figura $\mathrm{N}^{\circ} 7$

Variabilidad estacional en la composición de la macrofauna de crustáceos de la playa arenosa ubicada frente al revestimiento rocoso de Llico.

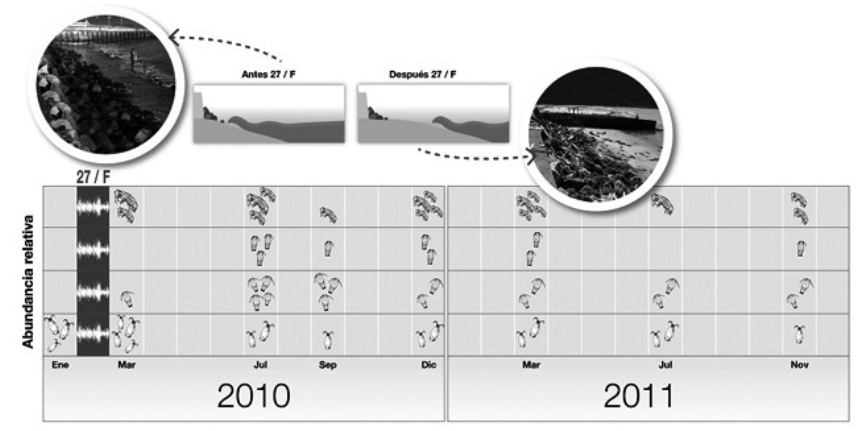

Fuente: Datos aportados por Eduardo Jaramillo. Esquema elaborado por Joaquín Sobell.

Figura $\mathrm{N}^{\circ} 8$

Variabilidad estacional en la composición de la macrofauna de crustáceos de la playa arenosa ubicada frente a la muralla de concreto de Lebu.

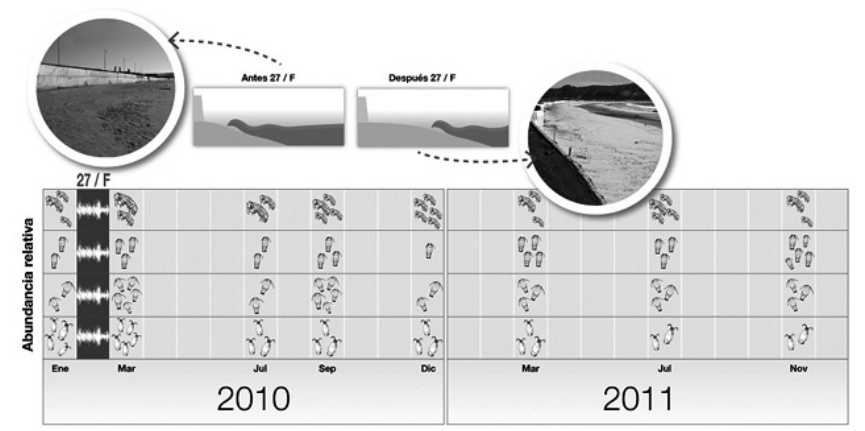

Fuente: Datos aportados por Eduardo Jaramillo. Esquema elaborado por Joaquín Sobell 


\section{Conclusión}

El ancho de la zona intermareal de la mayor parte de las playas de arena ubicadas en la península de Arauco, aumentó después de ocurrido el terremoto debido al levantamiento continental producto del mismo; coincidentalmente con esto, se observó una disminución de la pendiente o inclinación de las mismas. Esto es contrario a lo generalmente observado en áreas donde por efecto de un terremoto ocurre subducción costera, la que junto a los tsunamis resultantes producen erosión significativa de playas arenosas, tal como lo observado en playas de Tailandia, Sumatra y Japón después de los terremotos de los años 2004 y 2011 (Choowong et al., 2009; Liew et al., 2010; Udo et al., 2012).

Los cambios observados en el paisaje físico de las playas de arena inmediatamente después del 27F (marzo 2010), se mantuvieron durante los años 2010 y 2011 con perfiles de playa bastante similares después del mismo. Esto permite concluir que aun cuando el tsunami que siguió al terremoto removió enormes cantidades de arena, se suceden procesos que tienden a la estabilización de las playas, sometidas ahora a los procesos costeros que ocurren normalmente; por ejemplo, ciclos estacionales de erosión y acreción de arena. En general, los niveles inferiores de la zona intermareal de las playas de punta Lavapié, Llico y Lebu han ido aplanándose con el tiempo.

Los resultados de este estudio muestran que en playas donde con anterioridad al terremoto del $27 \mathrm{~F}$ había defensas costeras ubicadas cerca del nivel de marea alta y que fueron afectadas por el levantamiento continental, se restauró parte importante del espacio intermareal que con anterioridad a este evento se había perdido debido a la presencia de murallas de concreto y revestimientos rocosos. Dicho de otro modo los efectos de tal levantamiento sobre el ancho de la playa, fueron más notorios en aquellos sitios donde con anterioridad al 27F había mayor interacción con olas y mareas (punta Lavapie y Llico).

El espacio restaurado por efecto del levantamiento continental, comenzó a ser re- colonizado inmediatamente después del $27 \mathrm{~F}$ (marzo 2010) por especies de crustáceos que son típicas de los niveles superiores y medios de playas arenosas del centro-sur de Chile, carentes de defensas costeras artificiales. Esto demuestra que la baja diversidad de la macrofauna que ocurría en esas playas con anterioridad a este evento, era debido a que el espacio intermareal había sido ocupado por la instalación de murallas y revestimientos rocosos. Conclusiones similares a estas ya habían sido mencionadas para este litoral (Jaramillo 2011) y para playas arenosas de la costa de California (Dugan \& Hubbard, 2006; Dugan et al., 2008), donde la presencia de murallas de concreto y revestimientos rocosos es una característica común del paisaje litoral.

En definitiva, conclusiones como estas son relevantes para la planificación y desarrollo del borde costero y deben ser atendidas por los organismos encargados de esas materias. El proceder de tal modo, permite compatibilizar el desarrollo urbano de la costa con la estabilidad ambiental de los ecosistemas arenosos aledaños; situación particularmente importante en la reconstrucción de bordes costeros afectados por deformaciones costeras y tsunamis producidos por terremotos, como el que afectó a la costa de la península de Arauco el 27 de febrero de 2010. Este estudio también establece, que muestreos de terreno llevados a cabo luego de que las perturbaciones de gran escala han ocurrido, son relevantes para aumentar el conocimiento sobre las interacciones entre fenómenos naturales de gran escala y la infraestructura costera artificial, lo que también permite una mejor planificación del borde costero, aspecto no trivial en un país donde grandes terremotos y tsunamis son eventos recurrentes.

\section{Referencias bibliográficas}

BOGGS, K. \& SHEPHARD, M. Response of marine deltaic surfaces to major earthquake uplifts in southcentral Alaska. Wetlands, 1999, Vol. 19, № 1, p. 13-27.

BROWN, A. C. Biology of sandy beaches. In: STEELE, J. H.; THORPE, S. A. \& TUREKIAN, K. K. (Editors). Encyclopedia of Ocean Sciences. London: Academic Press, 2001, p. 2496-2504. 
CASTILLA, J. C. Earthquake-caused coastal uplift and its effects on rocky intertidal kelp communities. Science, 1988, Vol. 242, $\mathrm{N}^{\circ}$ 4877, p. 440-443.

CASTILLA, J. C.; MANRÍQUEZ, P. H. \& CAMAÑO, A. Effects of rocky shore coseismic uplift and the 2010 Chilean mega-earthquake on intertidal biomarker species. Marine ECO/ogy Progress Series, 2010, Vol. 418, p.17-23.

CHARLIER, R. H.; CHAINEUX, M. C. P. \& MORCOS, S. Panorama of the history of coastal protection. Journal of Coastal Research, 2005, Vol. 21, p. 79-111.

CHOOWONG, M.; PHANTUWONGRAJ, S.; CHAROENTITIRAT, T.; CHUTAKOSITKANON, V.; YUMUANG, S. \& CHARUSIRI, $P$. Beach recovery after 2004 Indian Ocean tsunami from Phang-nga, Thailand. Geomorphology, 2009, Vol. 104, p. 134-142.

DARWIN, C. Geological observations on coral reefs, volcanic islands and on South America. Londres: Smith, Elder and Co, 1851.

DUGAN, J. E. \& HUBBARD, D. M. Ecological responses to coastal armoring on exposed sandy beaches. Shore and Beach, 2006, Vol. 74, p. 10-16.

DUGAN J. E.; HUBBARD, D. M.; RODIL, I. F.; REVELL, D. L. \& SCHROETER, S. ECological effects of coastal armoring on sandy beaches. Marine Ecology: An evolutionary perspective, 2008, Vol. 29, p. 160 - 170.

EMERY, K. O. Simple method of measuring beach profiles. Limnology and Oceanography, 1961, Vol. 6, Nº 1, p. 90-93.

FARÍAS, M.; VARGAS, G.; TASSARA, A.; CARRETIER, S.; BAIZE, S.; MELNICK, D. \& BATAILLE, K. Land-level changes produced by the $2010 \mathrm{Mw}$ 8.8 Chile earthquake. Science, 2010 , p. 329.

GRIGGS, G. B. California's retreating coastline: where do we go from here? In: MAGOON, O. T.; CONVERSE, H.; BAIRD, B. \& MILLER-HENSON, M. (Editors). California and the World Ocean. Conference '02 Revisiting and Revising California's Ocean Agenda.
American Society of Civil Engineers, 2005a, p. 121-125.

GRIGGS, G. B. The impacts of coastal armoring. Shore and beach, 2005b, Vol. 73, p. $13-22$.

JARAMILLO, E. The sand beach ecosystem of Chile. In: SEELIGER, U. \& KJERFVE, B. (Editors). Coastal Marine Ecosystems of Latin America. Ecological Studies. Berlin: Springer Verlag, 2001, p. $219-227$.

JARAMILLO, E. Defensas costeras en playas arenosas de la región del Bío Bío y Maule: qué podemos aprender de su interacción con el terremoto del 27 de Febrero de 2010. Arquitecturas del Sur, 2011, Vol. 38, p. 66-79.

JARAMILLO, E.; DUGAN, J. E.; HUBBARD, D. M.; MELNICK, D.; MANZANO, M.; DUARTE, C.; CAMPOS, C. \& SÁNCHEZ, R. Ecological implications of extreme events: Footprints of the 2010 earthquake along the Chilean coast. PLOS ONE, 2012, Vol. 7, N 5, p. 1-8.

KRAUS, N. C. \& MCDOUGAL, W. G. The effects of seawalls on the beach: part 1, an updated literature review. Journal of Coastal Research, 1996, Vol. 12, p. 691-701.

LIEW,S.; GUPTA, A.; WONG, P. \& $\mathrm{KWOH}, \mathrm{L}$. Recovery from a large tsunami mapped over time: the Aceh coast, Sumatra. Geomorphology, 2010, Vol. 114, p. 520-529.

MCLACHLAN, A. \& JARAMILLO, E. Zonation on sandy beaches. Oceanography and Marine Biology: Annual Review, 1995, Vol. 33, p. 305-335.

NORDSTROM, K. F. Beaches and Dunes on Developed Coasts. Cambridge: Cambridge University Press, 2000.

ORTLIEB, L.; ZAZO, C.; GOY, J.L.; HILLAIRE-MARCEL, C.; GHALEB, B. \& COUMOYER, L. Coastal deformation and sea-level changes in the northern Chile subduction area $\left(23^{\circ} \mathrm{S}\right)$ during the last $330 \mathrm{KY}$. Quaternary Science Reviews, 1996a, Vol. 15, No 8, p. 819831(13). 
ORTLIEB, L.; BARRIENTOS, S. \& GUZMÁN, N. Coseismic coastal uplift and coraIline algae record in northern Chile: The 1995 Antofagasta earthquake case. Quaternary Science Reviews, 1996b, Vol. 15, p. 949-960.

PLAFKER, G. Tectonic deformation associated with the 1964 Alaska earthquake. Science, 1965, Vol. 148, №3678, p. 1675 1687.

PLAFKER, G. \& SAVAGE, J. C. Mechanism of the Chilean earthquake of May 21 and 22, 1960. Geological Society of America Bulletin, 1970, Vol. 81, p. 1001-1030.
UDO, K.; SUGAWARA, D.; TANAKA, H.; IMAI, K. \& MANO, A. Impact of the 2011 Tohoku earthquake and tsunami on beach morphology along the northern Sendai coast. Coastal Engineering Journal, 2012, Vol. 54, Nº 1, p. 1-15.

VARGAS, G.; FARÍAS, M.; CARRETIER, S.; TASSARA, A.; BAIZE, S. \& MELNICK, D. Coastal uplift and tsunami effects associated to the $2010 M_{w} 8.8$ Maule earthquake in central Chile. Andean Geology, 2011, Vol. 38, p. 219 - 238. 